\title{
The First Integral Method for Solving Exact Solutions of Two Higher Order Nonlinear Schrödinger Equations
}

\author{
Qingmei Zhang, Mei Xiong* and Longwei Chen \\ College of Statistics and Mathematics, Yunnan University of Finance and Economics, \\ Kunming, 650021, China \\ Email: 1477273038@qq.com, tc715@sin.com
}

\begin{abstract}
In this paper, exact travelling wave solutions of two higher order nonlinear Schrödinger equations (NLSEs) are studied by using the first integral method. Firstly, two higher order nonlinear Schrödinger equations are reduced to nonlinear ordinary differential equations (ODEs) by simple travelling wave transformations. Then the division theorem of polynomial is used to calculate first integrals of dynamic systems. Finally, the soliton wave solutions, kink wave solutions and periodic wave solutions of two higher order nonlinear Schrödinger equations are obtained. The results show that this method is effective for solving exact solutions of nonlinear partial differential equations (PDEs).
\end{abstract}

Keywords: The first integral method, the high order nonlinear Schrödinger equation, the exact travelling wave solutions.

\section{Introduction}

The nonlinear PDE is an important model for describing the problems of physics, chemistry, engineering and medicine. When we want to understand the principle of physical phenomena, the work to solve exact solutions of nonlinear PDEs and to research their properties is significative. In order to find the exact solutions of nonlinear PDEs, pioneers presented the following methods, such as tanh-sech function method [1], projective Riccati equation method [2], Kudryashov method [3], sine-cosine method [4], Jacobi elliptic function expansion method [5], F expansion method [6], exp-function method[7], Hirota bilinear method [8], bifurcation theory method of dynamic systems [9] and so on.

One of these methods is called the first integral method, which is based on the ring theory of commutative algebra. The integral method proposed by Feng [10] is reliable to solve nonlinear PDEs, and Feng further developed the theory by himself [11-13]. The theory has been applied to handle various PDEs by many scholars in science and engineering [14-16]. Here, we consider the higher order nonlinear Schrödinger equation with derivative non-Kerr nonlinear terms[17] and the higher order dispersive Cubic-quintic nonlinear Schrödinger equation [18]:

$$
\begin{gathered}
i E_{Z}-\frac{\beta_{2}}{2} E_{t t}+\gamma_{1}|E|^{2} E=i \frac{\beta_{3}}{6} E_{t t t}+i \alpha_{1}\left(|E|^{2} E\right)_{t} \\
+i \alpha_{2} E\left(|E|^{2}\right)_{t}-\gamma_{2}|E|^{4} E+i \alpha_{3}\left(|E|^{4} E\right)_{t}+i \alpha_{4} E\left(|E|^{4}\right)_{t},
\end{gathered}
$$

and

$$
i q_{Z}-\frac{\beta_{2}}{2} q_{t t}+\gamma_{1}|q|^{2} q-i \frac{\beta_{3}}{6} q_{t t t}-\frac{\beta_{4}}{24} q_{t t t t}+\gamma_{2}|q|^{4} q=0 .
$$

where $\alpha_{1}, \alpha_{2}, \alpha_{3}, \alpha_{4}, \beta_{2}, \beta_{3}, \beta_{4}, \gamma_{1}, \gamma_{2}$ are real constants. $E, q$ are complex functions.

For the higher order nonlinear Schrödinger equation with derivative non-Kerr nonlinear terms, Li et.al employed the bifurcation theory method of dynamical systems to provide the equation bounded traveling wave solutions [17]. Choudhuri and Porsezian analytically solved the high order NLSEs with non-Kerr nonlinearity under some parametric conditions, and he investigated explicitly bright and dark solitary wave solutions and periodic wave solutions [19]. In addition, other scholars have also used different methods to obtain the solutions of the equation [20-24]. For the higher order dispersive Cubic-quintic 
nonlinear Schrödinger equation, Zayed and Nowehy combined the solution Ansatz method with the Jacobi elliptic equation method to obtain several integrations denoted Jacobi elliptic function of the equation [25]. Arshad et.al used a modified extended direct algebraic method to present bright and dark wave solutions and soliton wave solutions of higher order dispersive Cubic-quintic NLSEs [26]. In addition, there are some papers [27-29] where the various types of higher-order NLSEs are studied.

The structure of this paper is organized as follows: Section 2 is the description of the basic idea of the first integral method briefly. In section 3, we use this method to solve two high order NLSEs in detail. In section 4 , some conclusions are given.

\section{The First Integral Method}

Consider the general nonlinear PDE in the form

$$
P\left(u, u_{t}, u_{x}, u_{x x}, u_{t t}, u_{x t}, u_{x x x}, \ldots\right)=0 .
$$

where $P$ is a polynomial in its arguments.

In order to transform the equation (3) into ODE, we introduce the transformation

$$
u(x, t)=u(\xi), \xi=x-c t .
$$

where $\mathrm{c}$ is a constant, then

$$
\frac{\partial}{\partial t}(\cdot)=-c \frac{\partial}{\partial \xi}(\cdot), \frac{\partial}{\partial x}(\cdot)=\frac{\partial}{\partial \xi}(\cdot), \frac{\partial^{2}}{\partial t^{2}}(\cdot)=c^{2} \frac{\partial^{2}}{\partial \xi^{2}}(\cdot), \ldots \ldots
$$

Step 1: According to above transformation, the equation(3) has the following nonlinear ODE form:

$$
Q\left(u, u_{\xi}, u_{\xi \xi}, \ldots \ldots\right)=0 .
$$

where the subscript denotes the derivation with respect to $\xi$.

Step 2: Suppose the solution of nonlinear ODE (6) can be written as

$$
u(x, t)=u(\xi) .
$$

Step 3: Let $X$ and $Y$ be a new independent variable

$$
X(\xi)=u(\xi), Y(\xi)=u_{\xi}(\xi)
$$

we obtain the system of ODEs

$$
\left\{\begin{array}{l}
X_{\xi}(\xi)=Y(\xi) \\
Y_{\xi}(\xi)=F(X(\xi), Y(\xi))
\end{array}\right.
$$

Step 4: According to the qualitative theory of ODEs [30], if we can find the first integrals to equation (9) under the same conditions, then the general solutions to equation (9) can be found directly. However, there is no systematic theory that can tell us how to find its first integrals, nor we know what these first integrals are. Thankfully, for some equations we can apply the Division Theorem to reduce equation (6) to a first order integrable ordinary differential equation. An exact solution to equation (3) is obtained by solving this equation. Now, let us review the Division Theorem:

Division Theorem: Suppose that $P(x, y)$ and $Q(x, y)$ are polynomials in $C(x, y)$, and make $P(x, y)$ is irreducible in $C(x, y)$. If $Q(x, y)$ vanishes at all zero points of $P(x, y)$, then there exists a polynomial $G(x, y)$ in $C(x, y)$, such that

$$
Q(x, y)=P(x, y) G(x, y)
$$




\section{Application}

In this section, we apply the first integral method based on the Division Theorem to solve the two higher order nonlinear Schrödinger equations.

Firstly, for equation (1), suppose the form of solution to equation (1) is as following:

$$
E(Z, t)=\psi(\xi) e^{i(k Z-w t)} .
$$

where $\xi=\beta Z-\lambda t$. And $\beta, \lambda, k, w$ are real parameters, $\psi(\xi)$ is a real function.

Substituting equation (11) into equation (1), and making real and imaginary part zero respectively:

$$
\begin{gathered}
\left(3 \lambda^{2} \beta_{2}+3 \lambda^{2} \beta_{3} w\right) \psi^{\prime \prime}-\left(\beta_{3} w^{3}+3 \beta_{2} w^{2}-6 k\right) \psi \\
-\left(6 \gamma_{1}-6 w \alpha_{1}\right) \psi^{3}-\left(6 \gamma_{2}+6 \alpha_{3} w\right) \psi^{5}=0 \\
\frac{1}{6} \beta_{3} \lambda^{3} \psi^{\prime \prime \prime}+\left(-\frac{1}{2} \beta_{3} w^{2} \lambda-\beta_{2} w \lambda+\beta\right) \psi^{\prime}+\left(3 \alpha_{1} \lambda\right. \\
\left.+2 \alpha_{2} \lambda\right) \psi^{2} \psi^{\prime}+\left(5 \alpha_{3} \lambda+4 \alpha_{4} \lambda\right) \psi^{4} \psi^{\prime}=0 .
\end{gathered}
$$

Integrating equation (13) once, clearly equation (13) and equation (12) have the following forms:

$$
\psi^{\prime \prime}-\frac{3 \beta_{3} w^{2} \lambda+6 \beta_{2} w \lambda^{2}-6 \beta}{\lambda^{3} \beta_{3}} \psi+\frac{6 \alpha_{1} \lambda+4 \alpha_{2} \lambda}{\lambda^{3} \beta_{3}} \psi^{3}+\frac{6 \alpha_{3} \lambda+\frac{24}{5} \alpha_{4} \lambda}{\lambda^{3} \beta_{3}} \psi^{5}=0 .
$$

where satisfy the constraint condition

$$
-\frac{3 \lambda^{2}\left(\beta_{2}+\beta_{3} w\right)}{\beta_{3} \lambda^{3} / 6}=\frac{\beta_{3} w^{3}+3 \beta_{2} w^{2}-6 k}{-(1 / 2) \beta_{3} w^{2} \lambda-\beta_{2} w \lambda+\beta}=\frac{6 \gamma_{1}-6 \alpha_{1} w}{3 \alpha_{1} \lambda+2 \alpha_{2} \lambda}=\frac{6 \gamma_{2}-6 \alpha_{3} w}{5 \alpha_{3} \lambda+4 \alpha_{4} \lambda} .
$$

Let $d_{1}=\frac{3 \beta_{3} w^{2} \lambda+6 \beta_{2} w \lambda^{2}-6 \beta}{\lambda^{3} \beta_{3}}, d_{2}=-\frac{6 \alpha_{1} \lambda+4 \alpha_{2} \lambda}{\lambda^{3} \beta_{3}}$ and $d_{3}=-\frac{6 \alpha_{3} \lambda+\frac{24}{5} \alpha_{4} \lambda}{\lambda^{3} \beta_{3}}$.

Putting equation (8) in equation (14), we get

$$
\left\{\begin{array}{l}
X^{\prime}(\xi)=Y(\xi) \\
Y^{\prime}(\xi)=d_{1} X(\xi)+d_{2} X(\xi)^{3}+d_{3} X(\xi)^{5}
\end{array}\right.
$$

Secondly, for equation (2). Suppose the form of solution to equation (2) is as following:

$$
q(Z, t)=\varphi(\xi) e^{i(\lambda Z-w t)}
$$

where $\xi=p Z-v t$. And $p, v, \lambda, w$ are real parameters, $\varphi(\xi)$ is a real function.

Substituting equation (17) into equation (2), and making imaginary and real part zero respectively:

$$
\begin{gathered}
\left(\beta_{3}-\beta_{4} w\right) v^{3} \varphi^{\prime \prime \prime}+\left(6 p-6 \beta_{2} v w-3 \beta_{3} v w^{2}+\beta_{4} v w^{3}\right) \varphi^{\prime}=0, \\
\beta_{4} v^{4} \varphi^{\prime \prime \prime \prime}+\left(-6 \beta_{4} v^{2} w^{2}+12 \beta_{3} v^{2} w+12 \beta_{2} v^{2}\right) \varphi^{\prime \prime}+\left(-12 \beta_{2} w^{2}+24 \lambda-4 \beta_{3} w^{3}+\beta_{4} w^{4}\right) \varphi \\
-24 \gamma_{1} \varphi^{3}-24 \gamma_{2} \varphi^{5}=0 .
\end{gathered}
$$

Differentiating equation (18) once, and substituting the resultant equation into equation (19), we make $k_{1}=\left(\beta_{3}-\beta_{4} w\right) v^{3}, k_{2}=6 p-6 \beta_{2} v w-3 \beta_{3} v w^{2}+\beta_{4} v w^{3}, k_{3}=-6 \beta_{4} v^{2} w^{2}+12 \beta_{3} v^{2} w+12 \beta_{2} v^{2}$ and $k_{4}=-12 \beta_{2} w^{2}+24 \lambda-4 \beta_{3} w^{3}+\beta_{4} w^{4}$. Then we can obtain 


$$
\varphi^{\prime \prime}-\frac{24 \gamma_{2} k_{1}}{k_{1} k_{3}-\beta_{4} v^{4} k_{2}} \varphi^{5}-\frac{24 \gamma_{1} k_{1}}{k_{1} k_{3}-\beta_{4} v^{4} k_{2}} \varphi^{3}+\frac{k_{4} k_{1}}{k_{1} k_{3}-\beta_{4} v^{4} k_{2}} \varphi=0 .
$$

Taking equation (8) in equation (20), we get

$$
\left\{\begin{array}{l}
X^{\prime}(\xi)=Y(\xi), \\
Y^{\prime}(\xi)=-\frac{k_{4} k_{1}}{k_{1} k_{3}-\beta_{4} v^{4} k_{2}} X(\xi)+\frac{24 \gamma_{1} k_{1}}{k_{1} k_{3}-\beta_{4} v^{4} k_{2}} X(\xi)^{3}+\frac{24 \gamma_{2} k_{1}}{k_{1} k_{3}-\beta_{4} v^{4} k_{2}} X(\xi)^{5} .
\end{array}\right.
$$

If we let again $d_{1}=-\frac{k_{4} k_{1}}{k_{1} k_{3}-\beta_{4} v^{4} k_{2}}, d_{2}=\frac{24 \gamma_{1} k_{1}}{k_{1} k_{3}-\beta_{4} v^{4} k_{2}}$ and $d_{3}=\frac{24 \gamma_{2} k_{1}}{k_{1} k_{3}-\beta_{4} v^{4} k_{2}}$, then equation (21) and (16) are equivalent to the first integral. In order to obtain their exact solution, we just need to talk about system equation (16).

Now, according to the first integral method. Assume that $X=X(\xi)$ and $Y=Y(\xi)$ are the nontrivial solutions of equation (16), and

$$
P(X, Y)=\sum_{i=0}^{m} a_{i}(X) Y^{i}
$$

is an irreducible polynomial in the complex domain $\mathrm{C}[\mathrm{X}, \mathrm{Y}]$ such that

$$
P(X(\xi), Y(\xi))=\sum_{i=0}^{m} a_{i}(X(\xi)) Y(\xi)^{i}=0
$$

where $a_{i}(X)(i=0,1, \ldots, m)$ are polynomial of $X$ and $a_{m}(X) \neq 0$. Equation (23) is called the first integral of the equation (16). Note that $P(X(\xi), Y(\xi))$ is a polynomial in $\mathrm{X}$ and $\mathrm{Y}$, and $\frac{d P}{d \xi}$ implies $\left.\frac{d P}{d \xi}\right|_{(22)}=0$. According to the division theorem, there exists a polynomial $H(X, Y)=h(X)+g(X) Y$ in $C(X, Y)$, that is,

$$
\left.\frac{d P}{d \xi}\right|_{(22)}=\left.\left(\frac{d P}{d X} \frac{d X}{d \xi}+\frac{d P}{d Y} \frac{d Y}{d \xi}\right)\right|_{(22)}=(h(X)+g(X) Y)\left(\sum_{i=0}^{m} a_{i}(X) Y^{i}\right) .
$$

Case 1: Assume that $m=1$ in equation (23) and then from equation (24) we have

$$
\sum_{i=0}^{1} a_{i}^{\prime}(X) Y^{i+1}+\sum_{i=0}^{1} i a_{i}(X) Y^{i-1}\left(Y^{\prime}(\xi)\right)=(h(X)+g(X) Y)\left(\sum_{i=0}^{1} a_{i}(X) Y^{i}\right) .
$$

where prime denotes differentiating with respect to the variable $X$. By comparing with the coefficient of $Y^{i}(i=2,1,0)$ on both sides of equation $(25)$, we have

$$
\begin{gathered}
a_{1}^{\prime}(X)=g(X) a_{1}(X), \\
a_{0}^{\prime}(X)=h(X) a_{1}(X)+g(X) a_{0}(X), \\
a_{1}(X)\left(d_{1} X+d_{2} X^{3}+d_{3} X^{5}\right)=h(X) a_{0}(X) .
\end{gathered}
$$

Since $a_{i}(X)(i=1,0)$ are polynomials, then from equation (26) we obtain that $a_{1}(X)$ is constant and $g(X)=0$. For simplicity, make $a_{1}(X)=1$. Balancing the degrees of $h(X)$ and $a_{0}(X)$, we get that $\operatorname{deg}(h(X))=2$ only. Assume that $h(X)=A X^{2}+B X+C$, where $A \neq 0$, then 


$$
a_{0}(X)=\frac{1}{3} A X^{3}+\frac{1}{2} B X^{2}+C X+D
$$

where $D$ is an arbitrary integration constant.

Substituting $a_{0}(X), a_{1}(X)$ and $h(X)$ into equation (28) and setting all the coefficient of powers of $\mathrm{X}$ equal to zero, we obtain a system of nonlinear algebraic equations, that is,

$$
\left\{\begin{array}{l}
\frac{1}{3} A^{2}=d_{3} \\
\frac{5}{6} A B=0 \\
\frac{4}{3} A C+\frac{1}{2} B^{2}=d_{2} \\
A D+\frac{3}{2} B C=0 \\
B D+C^{2}=d_{1} \\
C D=0
\end{array}\right.
$$

and using Maple solving them, we get

$$
D=0, B=0, A=\sqrt{3 d_{3}}, C=-\sqrt{d_{1}},
$$

and

$$
D=0, B=0, A=-\sqrt{3 d_{3}}, C=\sqrt{d_{1}},
$$

where $d_{1}, d_{3}<0$ satisfy the constraint condition

$$
d_{1} d_{3}=\frac{3}{16} d_{2}^{2}
$$

Taking the conditions (31) in (23), we get

$$
\left.Y(\xi)=X\left(-\frac{1}{3} \sqrt{3 d_{3}}\right) X^{2}+\sqrt{d_{1}}\right)
$$

and combining equation (33) with equation (16), we can obtain the exact solutions of the high order NLSE (1) as

$$
E_{1,2}(\xi)= \pm \frac{\sqrt{3 \sqrt{d_{1}} e^{\left(2 \sqrt{d_{1}} \xi\right)+\xi_{0}}}}{\sqrt{1-\sqrt{3 d_{3}} e^{\left(2 \sqrt{d_{1}} \xi\right)+\xi_{0}}}}
$$

where $\xi_{0}$ is an arbitrary constant. And the travelling wave solutions of the high order NLSE (1) can be written as

$$
E_{1,2}(Z, t)= \pm e^{i(k Z-w t)} \frac{\sqrt{3 \sqrt{d_{1}} e^{\left(2 \sqrt{d_{1}}(\beta Z-\lambda t)\right)+\xi_{0}}}}{\sqrt{1-\sqrt{3 d_{3}} e^{\left(2 \sqrt{d_{1}}(\beta Z-\lambda t)\right)+\xi_{0}}}} .
$$

Similarly, taking the conditions (32) in (23), we get

$$
\left.Y(\xi)=X\left(\frac{1}{3} \sqrt{3 d_{3}}\right) X^{2}-\sqrt{d_{1}}\right)
$$

from equation (16), we get 


$$
E_{3,4}(\xi)= \pm \frac{\sqrt{3 \sqrt{d_{1}}}}{\sqrt{e^{\left(2 \sqrt{d_{1}} \xi\right)+\xi_{0}}-\sqrt{3 d_{3}}}} .
$$

where $\xi_{0}$ is an arbitrary constant. And the travelling wave solutions of the high order NLSE (1) can be written as

$$
E_{3,4}(Z, t)= \pm e^{i(k Z-w t)} \frac{\sqrt{3 \sqrt{d_{1}}}}{\sqrt{e^{\left(2 \sqrt{d_{1}}(\beta Z-\lambda t)\right)+\xi_{0}}-\sqrt{3 d_{3}}}} .
$$

Case 2: Suppose that $m=2$, by comparing with the coefficient of $Y^{i}(i=3,2,1,0)$ on both sides of equation (24), we have

$$
\begin{gathered}
a_{2}^{\prime}(X)=g(X) a_{2}(X), \\
a_{1}^{\prime}(X)=h(X) a_{2}(X)+g(X) a_{1}(X), \\
a_{0}^{\prime}(X)+2 a_{2}(X)\left(d_{1} X+d_{2} X^{3}+d_{3} X^{5}\right)=h(X) a_{1}(X)+g(X) a_{0}(X), \\
a_{1}(X)\left(d_{1} X+d_{2} X^{3}+d_{3} X^{5}\right)=h(X) a_{0}(X) .
\end{gathered}
$$

Since $a_{i}(X)(i=2,1,0)=0$ are polynomials, then from equation (39) we obtain that $a_{2}(X)$ is a constant and $g(X)=0$. For simplicity, make $a_{2}(X)=1$. Balancing the degrees of $h(X), a_{1}(X)$ and $a_{0}(X)$, we get $\operatorname{deg}(h(X))=2, \operatorname{deg}\left(a_{1}(X)\right)=3, \operatorname{deg}\left(a_{0}(X)\right)=6$ only. Assume $h(X)=A X^{2}+B X+C$, where $A \neq 0$, then

$$
\begin{gathered}
a_{1}(X)=\frac{1}{3} A X^{3}+\frac{1}{2} B X^{2}+C X+D \\
a_{0}(X)=\left(\frac{1}{18} A^{2}-\frac{1}{3} d_{3}\right) X^{6}+\left(\frac{1}{6} A B\right) X^{5}+\left(\frac{1}{3} A C+\frac{1}{8} B^{2}-\frac{1}{2} d_{2}\right) X^{4} \\
+\left(\frac{1}{3} A D+\frac{1}{2} B C\right) X^{3}+\left(\frac{1}{2} B D+\frac{1}{2} C^{2}-d_{1}\right) X^{2}+(D C) X+E
\end{gathered}
$$

where $D$ is an arbitrary integration constant.

Substituting $a_{0}(X), a_{1}(X), a_{2}(X)$ and $h(X)$ into equation (42), setting all the coefficient of powers of $\mathrm{X}$ equal to zero, then we obtain a system of nonlinear algebraic equation, that is,

$$
\left\{\begin{array}{l}
\frac{1}{18} A^{3}=\frac{2}{3} A d_{3} \\
\frac{2}{9} A^{2} B=\frac{5}{6} B d_{3} \\
\frac{7}{18} A^{2} C+\frac{7}{24} A B^{2}=\frac{5}{6} A d_{2}+\frac{4}{3} C d_{3} \\
\frac{1}{3} A^{2} D+A B C+\frac{1}{8} B^{2}=B d_{2}+D d_{3} \\
\frac{7}{6} A B D+\frac{5}{6} A C^{2}+\frac{1}{8} B^{2} C=\frac{4}{3} A d_{1}+\frac{3}{2} C d_{2} \\
\frac{1}{2} D B^{2}+\frac{4}{3} A C D+B C^{2}=\frac{3}{2} B d_{1}+2 D d_{2} \\
\frac{3}{2} B C D+\frac{1}{2} C^{2}+A E=2 C d_{1} \\
D C^{2}+B E=D d_{1} \\
E C=0
\end{array}\right.
$$


and using Maple solving them, we obtain

$$
E=0, D=0, B=0, A=2 \sqrt{3 d_{3}}, C=-2 \sqrt{d_{1}},
$$

and

$$
E=0, D=0, B=0, A=-2 \sqrt{3 d_{3}}, C=2 \sqrt{d_{1}},
$$

where $d_{1}, d_{3}<0$ satisfy the constraint condition

$$
d_{1} d_{3}=\frac{3}{16} d_{2}^{2}
$$

Taking the conditions (45) in (23), we get

$$
\left.Y(\xi)=X\left(\frac{1}{6} \sqrt{3 d_{3}}\right) X^{2}-\frac{1}{2} \sqrt{d_{1}}\right),
$$

and combining equation (47) with equation (16), we can obtain the exact solutions of the high order NLSE (1) as

$$
E_{5,6}(\xi)= \pm \frac{\sqrt{3 \sqrt{d_{1}}}}{\sqrt{e^{\left(4 \sqrt{d_{1}} \xi\right)+\xi_{0}}-\sqrt{3 d_{3}}}} .
$$

where $\xi_{0}$ is an arbitrary constant. And the travelling wave solutions of the high order NLSE (1) can be written as

$$
E_{5,6}(Z, t)= \pm e^{i(k Z-w t)} \frac{\sqrt{3 \sqrt{d_{1}}}}{\sqrt{e^{\left(4 \sqrt{d_{1}}(\beta Z-\lambda t)\right)+\xi_{0}}-\sqrt{3 d_{3}}}} .
$$

Similarly, taking the conditions (46) in (23), we can obtain

$$
\left.Y(\xi)=X\left(-\frac{1}{6} \sqrt{3 d_{3}}\right) X^{2}+\frac{1}{2} \sqrt{d_{1}}\right)
$$

from equation(16), we have

$$
E_{7,8}(\xi)= \pm \frac{\sqrt{3 \sqrt{d_{1}} e^{\left(4 \sqrt{d_{1}} \xi\right)+\xi_{0}}}}{\sqrt{1-\sqrt{3 d_{3}} e^{\left(4 \sqrt{d_{1}} \xi\right)+\xi_{0}}}}
$$

Where $\xi_{0}$ is an arbitrary constant. And the travelling wave solutions of the high order NLSE (1) can be written as

$$
E_{7,8}(Z, t)= \pm e^{i(k Z-w t)} \frac{\sqrt{3 \sqrt{d_{1}} e^{\left(4 \sqrt{d_{1}}(\beta Z-\lambda t)\right)+\xi_{0}}}}{\sqrt{1-\sqrt{3 d_{3}} e^{\left(4 \sqrt{d_{1}}(\beta Z-\lambda t)\right)+\xi_{0}}}}
$$

\section{Conclusion}

The first integral method successfully solved some important nonlinear PDEs, and the method is implemented based on the ring theory of commutative algebra. Our works include two higher order NLSEs, and the soliton wave solutions, kink wave solutions and periodic wave solutions with different forms are obtained. Thus, for nonlinear PDEs, if we turn them into dynamic systems of five times by properly travelling wave transformations, then the same type solutions can be obtained by using the first integral method. Compared with other methods, it is an effective method to solve the exact traveling wave solution, that is, we can easily use computer software to complete the complex algebraic calculation, and more accurate traveling wave solution is obtained. Therefore, this method can be extended to solve other nonlinear PDEs. 


\section{References}

1. W.X.Ma, "Travelling wave solutions to a seventh order generalized KdV equation," Phys. Lett. A, 1993, (180), pp. 221-224.

2. R.Conte and M.Musette, "Link between solitary waves and projective Riccati equations," Journal of Physics A, 1992, pp. 5609-5623.

3. N.A.Kudryashov, "On types of nonlinear nonintegrable differential equations with exact solutions," Phys. Lett. A, 1991, (155), pp. 269-275.

4. A.M.Wazwaz, "A Sine-Cosine Method for Handling Nonlinear Wave Equations," Mathematical and Computer Modelling, 2004, (40), pp. 499-508.

5. S.Liu, Z.Fu, S.D.Liu and Q.Zhao, "Jacobi elliptic function expansion method and periodic wave solutions of nonlinear wave equations," Phys. Lett. A, 2001, (289), pp. 69-74.

6. S.Zhang, "The periodic wave solutions for the (2+1) dimensional Konopelchenko Dubrovsky equations," Chaos Solitons Fract, 2006, (30), pp. 1213-1220.

7. J.H.He and L.N.Zhang, "Generalized solitary solution and compacton-like solution of the Jaulent-Miodek equations using the Exp-function method," Physics Letters A, 2008, pp. 1044-1047.

8. D.S.Wang, "A systematic method to construct Hirotas transformations of continuous soliton equations and its applications," Computers and Mathematics with Applications, 2009, (58), pp. 146-153.

9. H.Wang, L.W.Chen, H.J.Liu and S.H.Zheng, "Nonlinear Dynamics and Exact Traveling Wave Solutions of the Higher-Order Nonlinear Schrödinger Equation with Derivative Non-Kerr Nonlinear Terms," Mathematical Problems in Engineering, vol. 2016, Article ID 7405141, 10 pages.

10. Z.S.Feng, "The first integral method to study the Burgers Korteweg-de Vries equation," J. Phys. A, 2002, (25), pp. $343-349$.

11. Z.S.Feng, "On explicit exact solutions to the compound Burgers Korteweg-de Vries equation," Phys. Lett.A, 2002, (293), pp. $57-66$.

12. Z.S.Feng, "Travelling wave behavior for a generalized Fisher equation," Soliton.Fract.A, 2008, (38), pp. 481-488.

13. Z.S.Feng, "Exact solution to an approximate sine-Gordon equation in (n+1)-dimensional space," Phys. Lett. A, 2002, (302), pp. 64-76.

14. S.Ibrahim and A.El-Ganaini, "The First Integral Method to the Nonlinear Schrödinger Equations in Higher Dimensions," Abstract and Applied Analysis, 2007,(18), pp. 1187-1197.

15. S.S.Singh, "Solutions of Kudryashov-Sinelshchikov equation and generalized Radhakrishnan-KunduLakshmanan equation by the first integral method," International Journal of Physical Research,2006, 4(2), pp. $37-42 .$.

16. A.Seadawy and A.Sayed, "Soliton Solutions of Cubic-Quintic Nonlinear Schrödinger and Variant Boussinesq Equations by the First Integral Method," Faculty of Sciences and Mathematics, 2017, (214), pp. 4199-4208.

17. Y.Li, W.R.Shan, T.P.Shuai and K.Rao, "Bifurcation Analysis and Solutions of a Higher-Order Nonlinear Schrödinger Equation," Mathematical Problems in Engineering, vol. 2015, Article ID 408586, 10 pages.

18. Y.Huang and P.Liu, "New Exact Solutions for a Class of High-order Dispersive Cubic-quintic Nonlinear Schröinger Equation," Canadian Center of Science and Education, 2014.

19. A.Choudhuri and K.Porsezian, "Higher-order nonlinear Schrödinger equation with derivative non-Kerr nonlinear terms: a model for sub-10-fs-pulse propagation," Physical Review A, 2013, (116), pp. 156-169.

20. V.K.Sharma, K.K.De and A.Goyal, "Solitary wave solutions of higher-order nonlinear Schrödinger equation with derivative non-Kerr nonlinear terms," Recent Advances in Photonics, 2014, pp. 1-2.

21. H.Triki, F.Azzouzi and P.Grelu, "Multipole solitary wave solutions of the higher-order nonlinear Schrödinger equation with quintic non-Kerr terms," Optics Communications, 2013, (309), pp. 71-79.

22. T.Houria and R.T.Thiab, "Solitary wave solutions for a higher order nonlinear Schrödinger equations," Mathematic Computers in Simulation, 2012, (82), pp. 1333-1340.

23. N.Taghizadeh, M.Mirzazadeh and F.Farahrooz, "Exact solutions of the nonlinear Schrödinger equation by the first integral method," J. Math. Anal. Appl, 2011, 37(4), pp. 549-553.

24. R.K.Raslan, "The first integral method for solving some im portant nonlinear partial differential equations," Nonlinear Dynam, 2008, 53(4), pp. 281-286.

25. EME. Zayed and AG-Al.Nowehy, "Exact solutions and optical soliton solutions for the nonlinear Schrödinger equation with fourth-order dispersion and cubic-quintic nonlinearity," Ricerche Di Matematica, 2017, pp. 1-22.

26. M.Arshad, A.R.Seadawy and D.Lu, "Elliptic function and solitary wave solutions of the higher-order nonlinear Schrödinger dynamical equation with fourth-order dispersion and cubic-quintic nonlinearity and its stability," European Physical Journal Plus, 2017, (132), pp. 371.

27. EME.Zayed and AG-Al.Nowehy, "Jacobi elliptic solutions, solitons and other solutions for the nonlinear Schrödinger equation with fourth-order dispersion and cubic-quintic nonlinearity," European Physical Journal Plus, 2017, (132), pp. 475. 
28. M.Arshad, A.R.Seadawy and D.Lu, "Exact bright dark solitary wave solutions of the higher-order cubic quintic nonlinear Schrödinger equation and its stability," Optik-International Journal for Light and Ele... , 2017, (138), pp. 40-49.

29. T.A.Davydova and Y.A.Zaliznyak, "Schrödinger ordinary solitons and chirped solitons: fourth-order dispersive effects and cubic-quintic nonlinearity," Physica D Nonlinear Phenomena, 2001, 156(34), pp. 260-282.

30. T.R.Ding and C.Z.Li, "Ordinary differential equations, peking:peking university press," 1996. 